\title{
The effect of serum proteins on dynamic interfacial properties of silicone oils in vitrectomized eyes
}

Irene Nepita ${ }^{1}$, Libero Liggieri ${ }^{3}$, Eva Santini ${ }^{3}$, Francesca Ravera ${ }^{3}$, Mario R. Romano², Jan O. Pralits ${ }^{1}$, Rodolfo Repetto ${ }^{1}$

${ }^{1}$ Department of Civil, Chemical and Environmental Engineering, University of Genoa, Genoa, Italy; 'Department of Biomedical Sciences, Humanitas University, Milan, Italy; ${ }^{3}$ Institute for Condensed Matter Chemistry and Energy Technologies, National Research Council, Genoa, Italy

\section{Abstract}

The formation and stability of emulsions in vitrectomized eyes is linked to the properties of the silicone oil-aqueous humor interface, in particular the surface tension. In the presence of natural surfactants, such as serum and plasma, the value of the surface tension is likely to change, but little quantitative information is presently available.

To this end, we perform accurate experiments measuring the interfacial properties of the Siluron 1000 (Fluoron GmbH, Ulm, Germany) silicone oil with an aqueous solution in the presence of endogenous-like proteins. It is found that the surface tension is significantly reduced when physiologically realistic concentrations are used. Moreover, the values obtained for the dilational viscoelastic modulus are compatible with the formation of stable emulsions.

Keywords: emulsion, silicone oil, surface tension, vitrectomy

\section{Introduction}

High-viscosity silicone oils (SO) are used in ophthalmology in a variety surgical procedures involving vitrectomy. This is the second most frequent surgical inter-

Correspondence: Irene Nepita, Department of Civil, Chemical and Environmental Engineering, University, of Genoa, Via Montallegro 1, 16145 Genoa, Italy.

E-mail: irene.nepita@gmail.com 
vention and is performed to treat several pathological or traumatic vitreoretinal conditions, such as retinal detachment, hemorrhage, inflammation, and infection in the eye.

The main complication associated with the use of SOs after vitrectomy is the tendency of the oil to emulsify in the aqueous solution produced in the eye, causing several postsurgical complications from moderate to serious. Hindering emulsification is therefore a mandatory step to improve the success of these surgical treatments.

The blood proteins produced by inflammatory processes have been identified as one of the co-factors responsible for the emulsification..$^{1-3}$ However, so far, the few available studies did not address the effects of these proteins on the key chemico-physical properties of the interface that are involved in the emulsion formation and stability. The present work is aimed at evaluating and characterizing the effects on the properties of the interface between $\mathrm{SO}$ and surfactant biomolecule aqueous solutions responsible for emulsification.

\section{Methods}

The interfacial tension (IT) and the interfacial dilational viscoelasticity (the response of interfacial tension to oscillatory perturbations of the interfacial area) have been measured at $35^{\circ} \mathrm{C}$ for the interface between SO and key serum proteins within the physiological concentration range in a Dulbecco alkaline buffer. ${ }^{4-5}$

The corresponding measurements have been performed in the proper characteristic time windows by using a Drop Shape tensiometer (PAT1; Sinterface, Berlin, Germany) that allows the control of the drop interfacial area. The shapes of liquid menisci result from the equilibrium between hydrostatic and capillary pressure at each point, according to the Young-Laplace Equation:

$$
\mathrm{P}_{0}+\Delta \rho g z=\gamma\left[\frac{1}{\mathrm{R}_{1}}+\frac{1}{\mathrm{R}_{2}}\right]
$$

where $R_{1}$ and $R_{2}$ are the curvature radii, $\gamma$ the interfacial tension, $P_{0}$ a constant, $\Delta \rho$ the density difference, and g gravity. This relation for the axisymmetric profile of a drop, as shown in Figure 1, can be redrawn by the Bashforth-Adams equation for an axisymmetric drop as follows:

$$
2+\frac{\beta z}{b}=\frac{1}{R / b}+\frac{\sin (\phi)}{x / b}
$$

where $b$ is the curvature radius at the apex, $R$ the curvature radius of the axial section at the point $P$ (Fig. 1), and $\beta$ the shape factor. The IT is obtained by the best fit of Equation (2) to the drop profile acquired from images of the drop. 


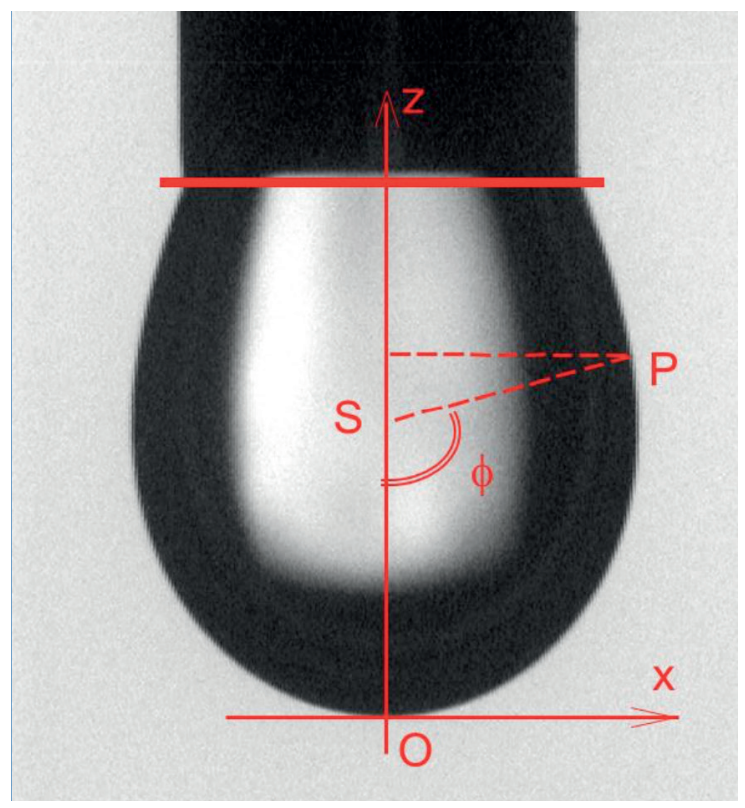

Fig. 1. Pendent drop with symbols used in Equation (2).

The dilational viscoelasticity, (Eq. (3) and Fig. 2), is measured according to the oscillating drop method, measuring the response of the IT to sinusoidal perturbations of the interfacial area, A. Here, $A 0$ is a reference area and $\varphi$ the phase.

$$
E=\frac{d \gamma}{d \ln A}=A 0 \frac{\Delta \gamma}{\Delta A} \exp (i \varphi)
$$

\section{Results}

The presence of endogenous proteins affects the interfacial properties remarkably. For the investigated albumin concentrations, Figure 3 reports the ratio between the equilibrium values of IT in the presence of albumin and those with the bare buffer. The ratio decreases to approximately 0.3 for an albumin concentration of $70 \mathrm{~g} / \mathrm{L}$, which most likely increases the tendency to emulsify. The time to attain equilibrium IT decreases from two hours to a few minutes by increasing albumin concentration.

Moreover, a typical serum protein in concentrations close to the physiological ones increases the dilational viscoelasticity modulus $(|\mathrm{E}|)$ to significantly higher values (Fig. 4), which are compatible with emulsions that, once formed, are expected to be more stable against coalescence. 


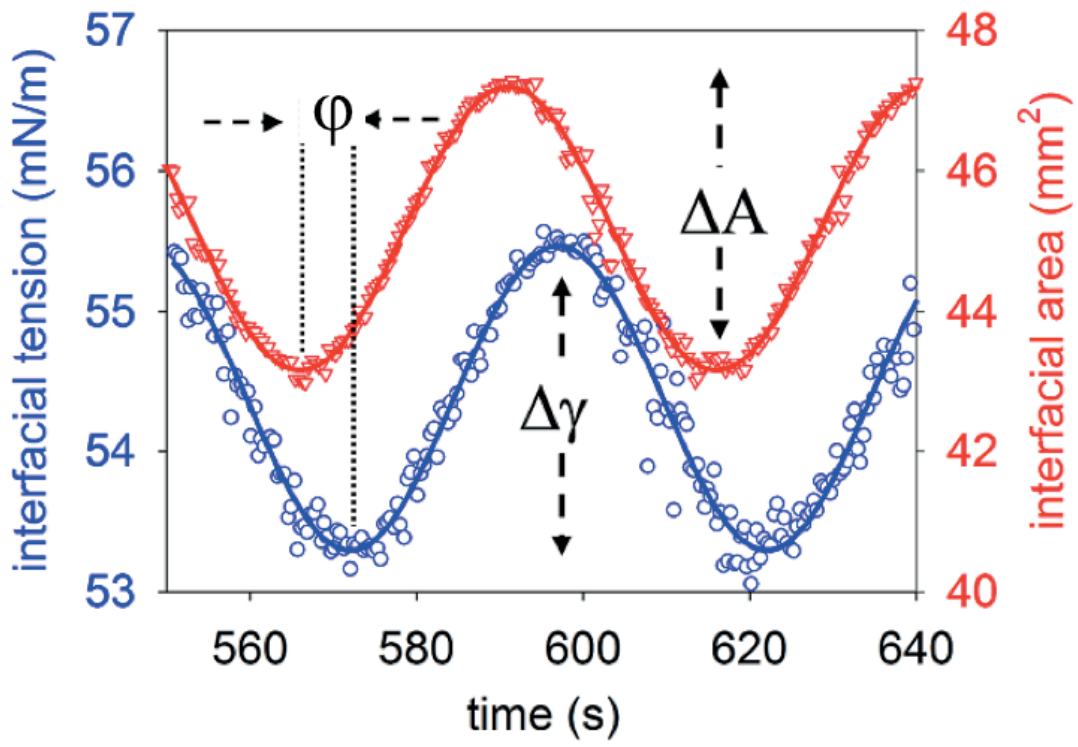

Fig. 2. Interfacial tension response to small amplitude harmonic perturbations of the interfacial area, A.

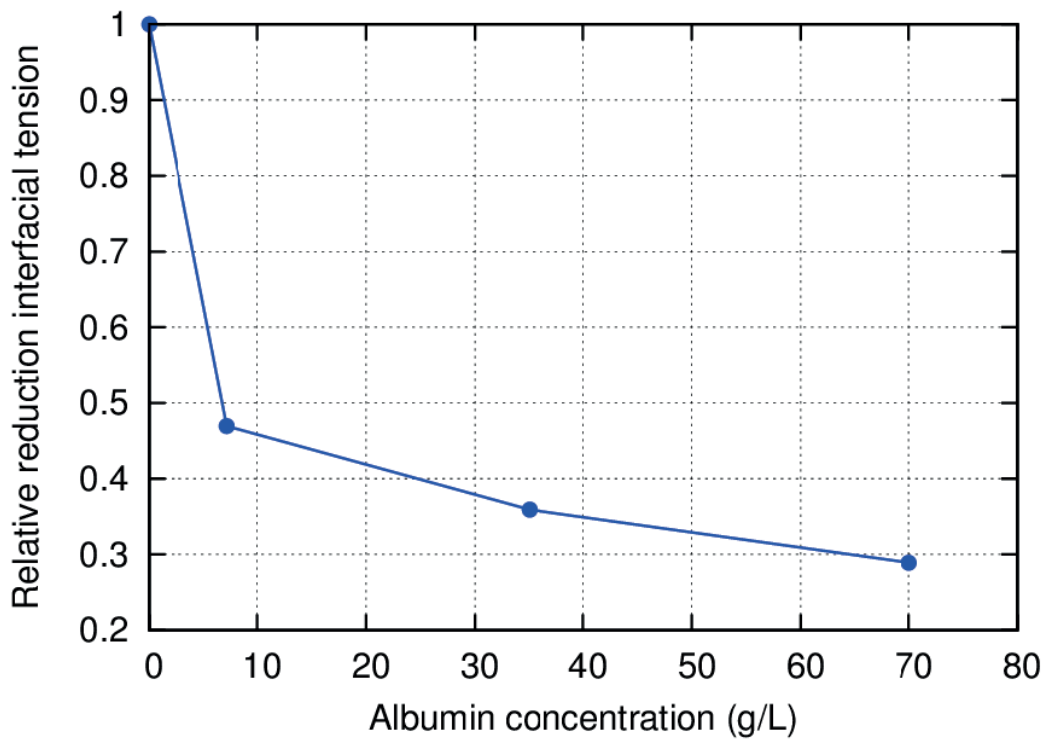

Fig. 3. The interfacial tension normalized with the value corresponding to pure SO-aqueous solution as a function of the albumin concentration. 


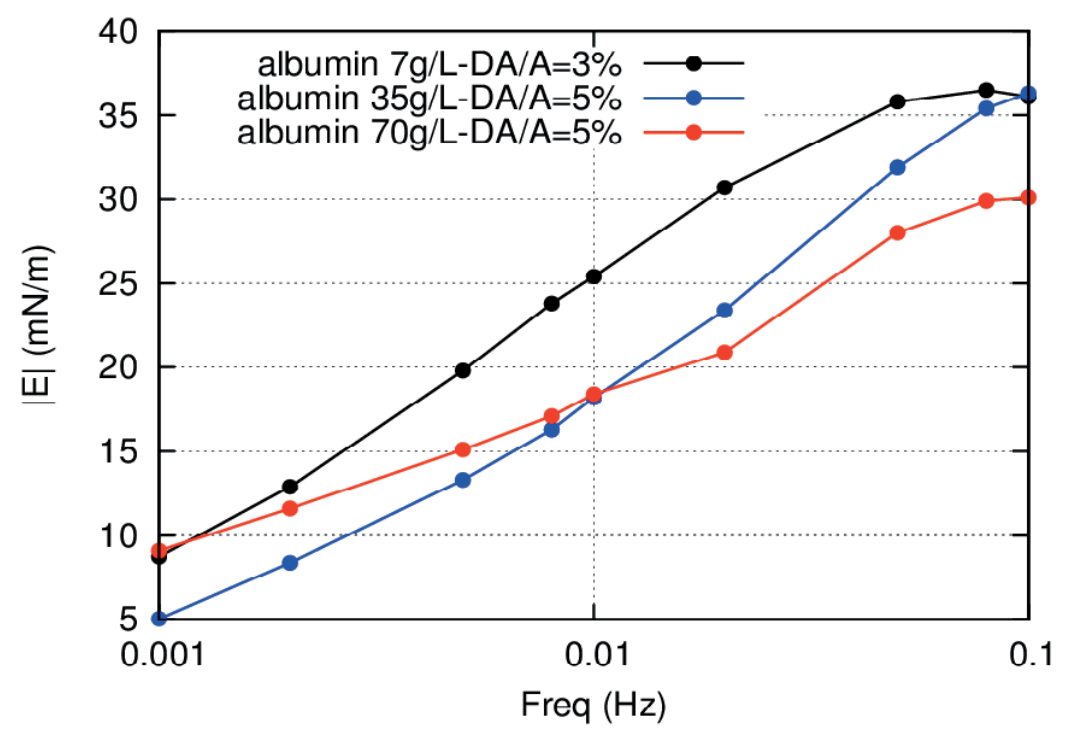

Fig. 4. Dilation viscoelasticity modulus at adsorption equilibrium measured as a function of the perturbation frequency for different albumin concentrations.

\section{Conclusions}

The adsorption of proteins at the liquid interface reduces the interfacial tension to values compatible with an increased tendency to emulsify and provides values of the dilational viscoelasticities compatible with a good stability for the resulting emulsions.

These observations are preliminary to design a wider study where different types of endogenous-like surfactants and their physiological blends will be investigated, suggesting possible strategies for the mitigation of the SO emulsification phenomena.

\section{Acknowledgements}

The silicone oil $1,000 \mathrm{mPa} / \mathrm{s}$ (molecular weight $37 \mathrm{kDa}$ ) tested in this study was Siluron 1000, kindly donated by Fluoron GmbH (Ulm, Germany). 


\section{References}

1. Nakamura K, Refojo MF, Crabtree DV. Factors contributing to the emulsification of intraocular silicone and fluorosilicone oils. Invest Ophthalmol Visual Sci.1990;31(4):647-656.

2. Caramoy A, Hagedorn N, Fauser S, Kugler W, Groß T, Kirchhof B. Development of emulsification-resistant silicone oils: can we go beyond $2000 \mathrm{mPas}$ silicone oil?. Invest Ophthalmol Visual Sci. 2011;52(8):5432-5436.

3. Zelisko PM, Flora KK, Brennan JD, Brook MA. Water-in-silicone oil emulsion stabilizing surfactants formed From native albumin and $\alpha, \omega$-triethoxysilylpropyl-polydimethylsiloxane. Biomacromolecules. 2008;9(8);2153-2161.

4. Loglio G, Pandolfini P, Miller R, Makievski AV, Ravera F, Ferrari M, Liggieri L. Novel methods to study interfacial layers. Stud Interface Sci. 2001;11:439.

5. Ravera F, Ferrari M, Santini E, Liggieri L. Influence of surface processes on the dilational visco-elasticity of surfactant solutions. Adv Colloid Interface Sci. 2005;117(1-3):75-100. 